\title{
Visión General Sobre el Proceso de Fitorremediación en Lodos Provenientes de Ptar
}

\author{
General Vision of Phytoremediation Treatment for \\ Sludge Derived of Wastewater
}

\author{
Yanneth Parra Martínez', Roberto Rafael Balda Ayala²
}

${ }^{1}$ Facultad de Ingeniería, Programa de Ingeniería Ambiental y Sanitaria, Universidad de La Salle, Bogotá D. C., Colombia. yparra@unisalle.edu.co

2 Facultad de Ingeniería, Programa de Ingeniería Ambiental y Sanitaria, Universidad de La Salle, Bogotá D. C., Colombia. rbalda@unisalle.edu.co

\section{Resumen}

Las aguas residuales provenientes de procesos industriales requieren tratamientos especiales que pueden ser de tipo físico-químico y/o biológico. El problema resultante de estos tratamientos lo constituyen los lodos generados como residuos, con contenidos de componentes de sustancias tóxicas entre ellas metales. En algunas empresas el problema que se presenta es la acumulación de los lodos en tanques en espera de proponer tratamientos o sistemas adecuados para su disposición; sin embargo, otras industrias sin responsabilidad ambiental se deshacen de ellos enviándolos al alcantarillado o a cuerpos superficiales de agua. Ante el panorama de la contaminación del agua y del subsuelo por una mala disposición de los lodos generados en plantas de tratamiento de aguas residuales, se desarrolló este proyecto en el que se caracterizaron y trataron lodos con alto contenido de metales pesados procedentes de tres plantas de tratamiento de aguas residuales: de una industria automotriz; de una industria de lácteos (lodos con contenido orgánico y graso), y de una población. Al comparar estos resultados con los requeridos en el Decreto 1594 de 1984, se observó que las concentraciones de los contaminantes eran mayores. Con base en esto, se efectuó un tratamiento de fitorremediación con dos especies de macrófitas. Durante la ejecución del proceso de fitorremediación, se verificó la migración de los contaminantes presentes en el lodo hacia alguna de las partes de la planta, con lo cual se comprobó la efectividad del proceso para la remoción de contaminantes de interés sanitario como cianuro, fenol, cinc y níquel.

Palabras clave: lodo orgánico, metales pesados, macrófitas, contaminantes.

\section{Abstract}

Wastewater coming from industrial processes requires special treatment either physical and/or biological. Their resulting problem is the sludge being produced, whose components are mainly inorganic with some organic content, and in the most unfavorable case, with the inclusion of toxic contents, i.e. heavy metals. In certain industries, the problem has to do with the accumulation of sludge in containers, before being disposed of in an appropriate way. However, sludge disposal by other non-environmentalist industries is carried out directly to the sewer or even, to water bodies. For developing this project, the water and soil pollution scope was taken into account, together with the poor disposal of sludge generated from wastewater treatment plants; hence, sludge with high heavy metal content and other pollutants coming from three wastewater treatment plants were characterized and treated: the first one, coming from an automobile industry; the second one, from a dairy industry (sludge with organic and fat content); and a third group coming from a municipality. When comparing the results of these analysis with the Colombian standards ( Decree 1594 of 1984), a higher pollution content was found. Therefore, a phytoremediation 
treatment was achieved with two species of microphytes. During the development of this process, pollutant migration was observed from the sludge toward one of the parts of the microphyte. Effectiveness of the process was tested for the removal of pollutants of sanitary interest: for example, cyanide, phenols, zinc, and nickel.

Keywords: organic sludge, heavy metals, microphytes, pollutants.

Recibido: 15/03/2011

Aceptado: 25/05/2011

\section{Introducción}

El proceso de fitorremediación consiste en la utilización de plantas (terrestres o acuáticas) con la capacidad fisiológica y bioquímica para absorber, retener, degradar o transformar, a formas menos tóxica, metales pesados, compuestos orgánicos, radioactivos y petro-derivados; a través de la utilización de cultivos in Vitro.

Las bases conceptuales de la fitorremediación provienen de la identificación de plantas que hiperacumulan metales. Existen plantas que tienen esta capacidad intrínseca, pero también pueden obtenerse plantas que adquieren esta capacidad por medio de técnicas de Ingeniería Genética.

Dentro de las ventajas de la fitorremediación se encuentran: las plantas pueden ser utilizadas para depurar suelos y aguas contaminadas; algunos procesos de degradación ocurren en forma más rápida con plantas que con microorganismos; es un método apropiado para descontaminar superficies grandes o finalizar la descontaminación de áreas restringidas en plazos largos.

En varios países como España y Argentina se han realizado estudios de fitorremediación especialmente en la recuperación de suelos. Por ejemplo, un ensayo de fitorremediación con girasol fue realizado por la Universidad de Córdoba en los suelos afectados por el vertido minero [1]. En este caso, los elementos más tóxicos ( $\mathrm{As}, \mathrm{Cd}, \mathrm{Pb}$ y $\mathrm{Tl}$ ), se acumularon preferentemente en la raíz. También se observó que el estrés del suelo contaminado, indujo que por cada gramo de $\mathrm{N}$ o $\mathrm{P}$ en hoja, se produjera mayor biomasa en la fase reproductora; mientras que por cada gramo de $\mathrm{Zn}$ o $\mathrm{Cd}$ (acumulados en exceso) se produjera menor biomasa. Los elementos traza se encontraron en la hoja y/o retenidos en la raíz, para que no interfirieran en el crecimiento y normal funcionamiento de la planta. Con esto se corroboraron los resultados de la presente investigación, donde la acumulación en las especies estudiadas se dio principalmente en la raíz, lo cual resulta interesante desde el punto de vista de utilización comestible de las mismas. Es así como, en el proyecto de grado de Tiria [2], quien trabajó con trigo y cebada, el cromo hexavalente de los lodos generados por la planta de tratamiento de aguas residuales de una industria de galvanotecnia, migró hacia la raíz y mantuvo el valor comestible de estas plantas.

Otros estudios, también enfocados hacia la recuperación de suelos mediante la fitorremediación han sido realizados en el Instituto de Investigaciones Agrobiológicas de Galicia [3] donde se ha trabajado en el desarrollo de chopos (Populus ligra) y abedules (Betula spp.) modificados genéticamente para limpiar suelos contaminados por metales pesados, como plomo o cadmio, y derivados de explosivos. El chopo y el abedul "son especies de crecimiento relativamente rápido, que están muy bien adaptadas a diferentes zonas de la geografía española y podrían aplicarse en la recuperación de terrenos contaminados a causa de actividades mineras o industriales", explica Antonio Ballester, investigador principal del proyecto.

En Argentina [4], se han llevado a cabo estudios con la Discaria americana la cual tiene la capacidad de acumular Zn, con destrucción de tejidos cuando los cultivos se efectúan en hidroponía con concentraciones de 40 a $100 \mathrm{mg} \mathrm{Zn/g;} \mathrm{esto} \mathrm{no} \mathrm{ocurrió} \mathrm{cuando} \mathrm{las} \mathrm{plantas} \mathrm{fueron} \mathrm{cultivadas} \mathrm{en}$ suelo. Las plantas extrajeron el $\mathrm{Zn}$ que se acumuló en sus raíces y al finalizar el ensayo alcanzó una concentración, respecto al testigo, de hasta 28 veces en las raíces y de hasta 5 veces en las parte aérea. Por otra parte, la concentración de nitrógeno en la planta no varió en forma significativa para las distintas concentraciones de $\mathrm{Zn}$ aplicadas al suelo.

El objetivo principal de este trabajo fue aplicar la fitorremediaciòn con lodos provenientes de plantas de tratamiento de vertimientos industriales en dos especies vegetales, procedentes del humedal Santa María del Lago ubicado en la ciudad de Bogotá (Colombia), con el fin de verificar la migración de contaminantes de interés sanitario tales como cianuro, fenol, cinc, níquel etc., hacia alguna zona de la planta. 


\section{Experimentación}

Para los ensayos de fitorremediación se utilizaron plantas acuáticas seleccionadas en cultivos in Vitro, adicionándoles cantidades conocidas de lodos con el fin de remover y contener los productos contaminantes presentes en él.

Se utilizaron dos especies vegetales, el Biden leavis (botoncillo flora amarilla) y Pteridium aquilinum (Cytheatae), las cuales fueron seleccionadas después de un proceso de pre-experimentación que consistió en determinar el grado de adaptación de las especies Lemna (lenteja de agua), el Scirpus californicus (Junco), la subclase Cyatheatae (helecho), con la especie Pteridium aquilinum,; el Biden leavis (botoncillo flora amarilla) y Typha latifolia (Tifa), provenientes del Humedal Santa María del Lago. El Humedal Santa María del Lago, pertenece a la localidad de Engativá situada en la ciudad de Bogotá, este humedal cuenta con la capacidad de almacenar agua en forma permanente y recibe un aporte hídrico continuo de aguas freáticas y lluvias; estas características le permiten cumplir con sus funciones de nicho ecológico, además de actuar como filtro purificador de las aguas residuales provenientes de algunos barrios aledaños.

La experimentación fue desarrollada en el Laboratorio de Planta Piloto del Programa de Ingeniería Ambiental y Sanitaria de la Universidad de La Salle en Bogotá; allí se realizó el montaje con una repetición para cada especie; dicho montaje consistió en sembrar las plantas en materas donde se agregó para su soporte grava malla referencia 6-12 y grava de malla referencia 12-20, $1000 \mathrm{ml}$ de agua del humedal y $100 \mathrm{~g}$ de lodo en cada una de las materas. A su vez, diariamente se adicionaban $100 \mathrm{ml}$ de agua del humedal, con la finalidad de mantener la cantidad de agua en la matera dado que se presentaban perdidas por procesos de evaporación.

Para cada uno de los lodos estudiados, durante la pre-experimentación se realizaron 48 ensayos para las 6 especies vegetales mencionadas anteriormente (8 para cada especie) con la distribución que se presenta en la tabla 1.

Tabla 1 Matriz pre-experimental para cada especie

\begin{tabular}{|l|c|c|l|}
\hline ESPECIE & TRATAMIENTO & No. REPETICIÓNES & $\begin{array}{l}\text { TOTAL } \\
\text { TRATAMIENTOS } \\
\text { POR ESPECIE }\end{array}$ \\
\hline \multirow{3}{*}{ Lemma (lenteja de agua) } & $1-$ Lodo 1 & 1 & \multirow{2}{*}{8} \\
\cline { 2 - 3 } & $2-$ Lodo 2 & 1 & \\
\cline { 2 - 3 } & $3-$ Lodo 2 & 1 & \\
\cline { 2 - 3 } & $4-$ Lodo & 1 & \\
\hline
\end{tabular}

Fuente: Autores, 2005

Las condiciones del experimento fueron: temperatura ambiente promedio $\left(17-18^{\circ} \mathrm{C}\right)$ y Presión del laboratorio de $557.7 \mathrm{mmHg}$.

Los lodos seleccionados para los tratamientos se presentan en la tabla 2.

Tabla 2 Clasificaciyn de lodos

\begin{tabular}{|l|c|c|}
\hline PROCEDENCIA & TIPO DE LODO & CLASIFICACIÓN \\
\hline $\begin{array}{l}\text { PTAR de metales pesados de una } \\
\text { Industria Automotriz }\end{array}$ & Con alto contenido metálico & 1 \\
\hline $\begin{array}{l}\text { PTAR general de una Industria } \\
\text { Automotriz }\end{array}$ & $\begin{array}{c}\text { Ligero contenido metálico y orgánico y de } \\
\text { residuos de pinturas }\end{array}$ & 3 \\
\hline PTAR de una Municipalidad & Material orgánico & 3 \\
\hline PTAR de una Industria Alimenticia & Material orgánico y graso & 4 \\
\hline
\end{tabular}


A cada uno de los lodos se le realizó el análisis químico de 22 parámetros (con su respectiva repetición), el análisis se encuentra relacionado con el promedio de resultados en la tabla 3.

Tabla 3 Anбlisis de lodos

\begin{tabular}{|l|l|l|l|l|}
\hline DETERMINACIÓN & LODO1 & LODO 2 & LODO 3 & LODO 4 \\
\hline Ph & 8.07 & 7.59 & 6.3 & 5.97 \\
\hline Conductividad ms/cm & 0.685 & 0.81 & 2.6 & 0.755 \\
\hline Nitritos m g/L & 0.05 & 0.1 & 2.5 & 0.0 \\
\hline Nitratos m g/L & 5 & 8 & 22.5 & 2 \\
\hline Amonio m g/L & 2 & 2 & 0.6 & 0.4 \\
\hline Cianuro m g/L & 0.01 & 0.03 & 0.12 & 0.055 \\
\hline Fenol $\mathrm{m} \mathrm{g/L}$ & 0.7 & 3.45 & 0.1 & 0.15 \\
\hline Fosfatos m g/L & 185 & 24 & 148 & 122 \\
\hline Níquel $\mathrm{m} \mathrm{g/L}$ & 0.95 & 1.3 & 0.1 & 0.1 \\
\hline Arsénico m g/L & 0.0 & 0.0 & 0.07 & 0.0 \\
\hline Sodio $\mathrm{m} \mathrm{g/L}$ & 136.25 & 11.16 & 6.1 & 7.68 \\
\hline Calcio $\mathrm{m} \mathrm{g/L}$ & 26.58 & 189.52 & 15.56 & 83.7 \\
\hline Hierro m g/L & 715.02 & 217.3 & 59.9 & 24.13 \\
\hline Cobre m g/L & 92.75 & 182.16 & 40.36 & 5.05 \\
\hline Plomo m g/L & 184.24 & 4.88 & 47.72 & 26.1 \\
\hline Cinc m g/L & 9.54 & 454.4 & 53 & 16.9 \\
\hline Nitrógeno total m g/L & 7.0 & 10.1 & 4.07 & 3.9 \\
\hline Magnesio m g/L & 9.14 & 7.49 & 1.10 & 4.9 \\
\hline Potasio m g/L & 82.95 & 9.28 & 11.28 & 9.69 \\
\hline \% Humedad & 65 & 77 & 80 & 75 \\
\hline Cromo m g/L & 121.1 & 209.3 & 50.4 & 31.75 \\
\hline Aceites y grasas m g/L & 0.0 & 0.0 & 1.19 & 331.65 \\
\hline \hline
\end{tabular}

Fuente: Autores, 2005

La prueba se efectuó por un periodo de 8 días durante los cuales se verificó el grado de adaptación de cada una de las especies vegetales en presencia de las muestras de lodos. Allí se observó que las especies Biden leavis (botoncillo flora amarilla) y Pteridium aquilinum (Cytheatae), se seleccionaron porque presentaron el mejor comportamiento en cuanto a durabilidad, frescura, coloración verde de la planta y resistencia al tratamiento.

Con base en los resultados de la fase pre-experimental, se planteó un diseño experimental en el que se trató cada especie por duplicado con cada uno de los lodos; se efectuaron 2 ensayos (con una réplica por especie) por cada lodo, de donde resultó un total de 16 unidades experimentales, como se presenta en la tabla 4. Los montajes se controlaron a diario, observando el comportamiento de la planta en cuanto a su crecimiento y procesos de envejecimiento y descomposición. Después de 5 días se inició la toma de muestra que incluyó las siguientes partes de la planta y los elementos que hicieron parte del ensayo: hojas, raíz y tallo, así como el agua residual lixiviada y el lodo residual. Estas muestras se analizaron en el Laboratorio Central de Ingeniería Ambiental y Sanitaria de la Universidad de La Salle, utilizando métodos fotométricos Nanocolor que son procedimientos reconocidos como una alternativa 
a los métodos estándares alemanes DIN. Es importante anotar que antes de las pruebas, también se analizó cada uno de los lodos, el agua del humedal de donde provenían las especies y las 2 gravas utilizadas en la siembra de la planta.

Tabla 4 Matriz experimental

\begin{tabular}{|c|c|c|c|}
\hline ESPECIES & \multicolumn{2}{|c|}{ TRATAMIENTO } & REPETICIÓN \\
\hline \multirow[t]{4}{*}{ Biden leavis (botoncillo flora amarilla) } & 1. & Lodo 1 & 1 \\
\hline & 2. & Lodo 2 & 1 \\
\hline & 3. & Lodo 3 & 1 \\
\hline & 4. & Lodo 4 & 1 \\
\hline \multirow[t]{4}{*}{ Pteridium aquilinum (Cytheatae) } & 1. & Lodo 1 & 1 \\
\hline & 2. & Lodo 2 & 1 \\
\hline & 3. & Lodo 3 & 1 \\
\hline & 4. & Lodo 4 & 1 \\
\hline
\end{tabular}

Fuente: Autores, 2005

Finalmente, después del tratamiento se realizó el análisis químico de 18 parámetros, con su respectiva réplica a cada una de las partes de la planta (raíz, tallo y hoja) para un total de 108 análisis.

\section{Resultados}

"La fitorremediación (phyto= planta y remediación = mal por corregir), es un proceso que utilizan plantas para remover, transferir, estabilizar, concentrar y/o destruir contaminantes (orgánicos e inorgánicos) en suelos, lodos y sedimentos, y puede aplicarse tanto in situ como ex situ" [5]. Durante la fase de experimentación, se apreció la ocurrencia de 2 categorías de fitorremediación expuestas en la literatura [6].

1. La rizofiltración: en esta categoría, se clasifican las plantas que concentran y absorben principalmente metales y compuestos orgánicos en las raíces de las plantas.

2.La fitoextracción: en esta categoría, se clasifican las plantas que concentran metales en las partes aéreas de la planta como el tallo y las hojas.

Para evaluar el porcentaje final de cada elemento en cada una de las partes de la planta, se realizó un balance de masa con base en la siguiente ecuación:

$$
\% \text { Absorbido }(R, H o T)=\frac{\text { Contaminante final }- \text { contaminante inicial }}{\text { Cont.adicionado }(\text { lodo }+ \text { grava }+ \text { agua humedal })} \times 100
$$

Siendo: $\mathrm{R}$ = raíz; $\mathrm{T}=$ Tallo; $\mathrm{H}$ = Hoja. Los resultados se muestran en la tabla 5. 
Tabla 5 Comportamiento de las especies Pteridium aquilinum (Cytheatae) y Beaden leavis durante el tratamiento de lodos

\begin{tabular}{|c|c|c|c|c|c|c|c|}
\hline \multicolumn{2}{|l|}{ LODO 1} & \multicolumn{2}{|l|}{ LODO 2} & \multicolumn{2}{|l|}{ LODO 3} & \multicolumn{2}{|l|}{ LODO 4} \\
\hline \multicolumn{8}{|c|}{ Elementos absorbidos por la especie Cytheatae } \\
\hline Elemento & $\begin{array}{l}\text { Sección } \\
\text { de planta }\end{array}$ & Elemento & $\begin{array}{l}\text { Sección de } \\
\text { planta }\end{array}$ & Elemento & $\begin{array}{l}\text { Sección } \\
\text { de planta }\end{array}$ & Elemento & $\begin{array}{l}\text { Sección de } \\
\text { planta }\end{array}$ \\
\hline \multirow[t]{2}{*}{$\mathrm{Ca}$} & R $89.6 \%$ & $\mathrm{CN}^{-}$ & R 33.3\% & $\mathrm{CN}^{-}$ & $\begin{array}{l}\text { Todas } \\
22.4 \%\end{array}$ & K & R-H 45.7\% \\
\hline & T 25.75\% & Fenol & R $2.7 \%$ & $\mathrm{~N}$ total & $\begin{array}{l}\text { Todas } \\
57 \%\end{array}$ & $\mathrm{PO}_{4}^{-3}$ & R-T-H 21\% \\
\hline $\mathrm{CN}^{-}$ & $\begin{array}{l}\text { Todas } \\
24 \% \\
\end{array}$ & $\mathrm{Na}$ & R $13.4 \%$ & $\mathrm{PO}_{4}^{-3}$ & $\begin{array}{l}\text { Todas } \\
28 \% \\
\end{array}$ & $\mathrm{CN}^{-}$ & Todas $33.3 \%$ \\
\hline $\mathrm{PO}_{4}^{-3}$ & $\begin{array}{c}\text { Todas } \\
28.02 \%\end{array}$ & $\mathrm{ZN}$ & R 38\% & Fenol & R $49.3 \%$ & $\mathrm{NI}$ & Todas $6.57 \%$ \\
\hline$N$ & $\begin{array}{l}\text { Todas } \\
57.14 \%\end{array}$ & $\mathrm{Mg}$ & R 25\% & $\mathrm{Ni}$ & R-H 4.3\% & $\mathrm{Mg}$ & T-H $18.65 \%$ \\
\hline \multirow[t]{2}{*}{ Fenol } & R $49.3 \%$ & $\mathrm{PO}_{4}^{-3}$ & R-H $51.6 \%$ & $\mathrm{Mg}$ & $\begin{array}{c}\mathrm{R}-\mathrm{H} \\
17.4 \% \\
\end{array}$ & Fenol & R-T 3.35\% \\
\hline & T 29.87 & $\mathrm{Ca}$ & Todas $47 \%$ & $\mathrm{~K}$ & R-H 62\% & & \\
\hline $\mathrm{Ni}$ & R $4.3 \%$ & $\mathrm{Fe}$ & $\begin{array}{l}\text { Todas } \\
43.8 \% \\
\end{array}$ & & & & \\
\hline \multirow[t]{2}{*}{$\mathrm{Mg}$} & H $17.4 \%$ & $\mathrm{Cu}$ & R-T 55\% & & & & \\
\hline & & $\mathrm{K}$ & R-T $67 \%$ & & & & \\
\hline \multicolumn{2}{|l|}{\begin{tabular}{|l|} 
LODO 1 \\
\end{tabular}} & \multicolumn{2}{|l|}{ LODO 2} & \multicolumn{2}{|l|}{ LODO 3} & \multicolumn{2}{|l|}{ LODO 4} \\
\hline \multicolumn{8}{|c|}{ Elementos absorbidos por la especie Biden Leváis } \\
\hline Elemento & $\begin{array}{l}\text { Sección } \\
\text { de planta } \\
\end{array}$ & Elemento & $\begin{array}{l}\text { Sección } \\
\text { de planta }\end{array}$ & Elemento & $\begin{array}{l}\text { Sección } \\
\text { de planta }\end{array}$ & Elemento & $\begin{array}{c}\text { Sección de } \\
\text { planta }\end{array}$ \\
\hline $\mathrm{CN}^{-}$ & T 75\% & $\mathrm{CN}^{-}$ & R-T 33.3\% & $\mathrm{CN}$ & $\begin{array}{c}\mathrm{T}-\mathrm{H} \\
45.4 \% \\
\end{array}$ & $\mathrm{PO}_{4}^{-3}$ & R $94 \%$ \\
\hline Fenol & $\begin{array}{c}\mathrm{R}-\mathrm{T} \\
16.6 \%\end{array}$ & $\begin{array}{l}\text { Fenol } \\
\text { Fosfatos }\end{array}$ & R-T $37 \%$ & Fenol & $\begin{array}{c}\mathrm{R}-\mathrm{H} \\
17.9 \%\end{array}$ & $\mathrm{Ca}$ & R $44.7 \%$ \\
\hline $\mathrm{PO}_{4}^{-3}$ & $\begin{array}{l}\text { Todas } \\
90 \%\end{array}$ & $\mathrm{Ni}$ & R-T $45.6 \%$ & PO4-3 & $\begin{array}{c}\text { Todas } \\
85 \%\end{array}$ & $\mathrm{Zn}$ & R $27.5 \%$ \\
\hline K & $\begin{array}{c}\text { Todas } \\
14.56 \% \\
\end{array}$ & $\mathrm{~Pb}$ & R-T $48 \%$ & $\mathrm{Ni}$ & $\begin{array}{c}\mathrm{R}-\mathrm{H} \\
16.75 \% \\
\end{array}$ & $\mathrm{Mg}$ & R $66.1 \%$ \\
\hline $\mathrm{Ni}$ & R $71 \%$ & $\mathrm{PO}_{4}^{-3}$ & T $87 \%$ & $\mathrm{Ca}$ & R $61 \%$ & & \\
\hline $\mathrm{Ca}$ & R $61 \%$ & $\mathrm{Na}$ & $\begin{array}{l}\text { Todas } \\
46.5 \% \\
\end{array}$ & $\mathrm{~N}$ total & R 40\% & & \\
\hline $\mathrm{Mg}$ & H 25.3\% & $\mathrm{Cr}$ & $\begin{array}{c}\text { Todas } \\
76.35 \% \\
\end{array}$ & & & & \\
\hline \multirow[t]{6}{*}{$\mathrm{Cr}$} & R 13\% & $\mathrm{Fe}$ & $\begin{array}{l}\text { Todas } \\
55.8 \% \\
\end{array}$ & & & & \\
\hline & & $\mathrm{Cu}$ & R-H 50\% & & & & \\
\hline & & $\mathrm{Zn}$ & $\mathrm{R}-85 \%$ & & & & \\
\hline & & $\mathrm{K}$ & R-43\% & & & & \\
\hline & & $\mathrm{NH}_{4}^{-}$ & $\mathrm{R}-50 \%$ & & & & \\
\hline & & $\mathrm{Mg}$ & H 64.8\% & & & & \\
\hline \multicolumn{8}{|l|}{$\mathrm{R}=$ Raiz } \\
\hline \multicolumn{8}{|l|}{$\mathrm{H}=$ Hojas } \\
\hline $\mathrm{T}=$ Tallo & & & & & & & \\
\hline
\end{tabular}




\section{Discusión}

De acuerdo con lo observado en la presente investigación se concluye que la especie Biden Leavis, presentó inmovilización de contaminantes metálicos y fenol apreciablemente en la sección de la raíz; especialmente en el ensayo efectuado con el lodo 4 procedente de la PTAR de alimentos. En otros estudios realizados en manglares, se encontró que:

Las concentraciones observadas en los metales analizados pusieron en evidencia que el plomo es el metal con mayor concentración en diferentes componentes del ecosistema el manglar. Los metales plomo, cromo y cadmio presentan una alta concentración en el sedimento, el cual actúa como una trampa que facilita el depósito de metales pesados en el manglar cuando se precipita en las inmediaciones de la raíz. Las raíces de especies de mangle, principalmente Laguncularia racemosa y A germinans, desempeñan un papel principal como biofiltros naturales en la absorción de metales pesados, seguidos por los troncos y las hojas [7].

Se probó además, que la fitorremediación es una técnica ecotecnológica apropiada para el manejo de lodos procedentes de plantas de tratamiento de aguas residuales, tanto domésticas como industriales, toda vez que sus resultados en absorción de contaminantes de interés sanitario, como lo son los fenoles y metales como $\mathrm{Pb}, \mathrm{Ní}, \mathrm{Mg}, \mathrm{Cu}, \mathrm{Fe}$, y $\mathrm{Zn}$ que se analizaron para el presente proyecto, fueron satisfactorios. Tal como se ha concluido en otro estudio sobre fitorremediación [8]. La fitorremediación puede aplicarse eficientemente para tratar suelos contaminados con compuestos orgánicos como benceno, tolueno, etilbenceno y xilenos (BTEX); solventes clorados; HAPs; desechos de nitrotolueno; agroquímicos clorados y organofosforados; además de compuestos inorgánicos como Cd, Cr(VI), Co, Cu, Pb, Ni, Se y Zn. Se Ha demostrado también su eficiencia en la remoción de metales radioactivos y tóxicos de suelos y aguas[8].

Frente a otras técnicas de remoción como las de precipitación u oxidación química, una de las ventajas principales de la fitorremediación es la disminución del volumen de lodos a disponer al final. En este caso, la disposición de residuos es mucho menor, limitándose a las partes colmatadas de la planta correspondiente. De acuerdo con Agudelo, los metales absorbidos por las plantas, pueden ser extraídos de la biomasa cosechada y después se reciclados. La fitorremediación se puede utilizar para limpiar una gran variedad de contaminantes, por su capacidad de absorción de estos metales en sus raíces. Para reducir la entrada de contaminantes en el ambiente, disminuyendo su salida a los sistemas de aguas subterráneas, porque los contaminantes quedan atrapados en las plantas [9].

De acuerdo con los resultados obtenidos, se observa que la mayor acumulación de contaminantes se presentó en la zona de la raíz seguida del tallo y, finalmente, la menor en las hojas, esto puede deberse a que para algunos tipos de planta procedentes de humedales, algunos estudios [10] sobre la aplicación de sistemas para fitorremediación en suelos contaminados por metales pesados en la zona de AznalcóllarEspaña demostraron que la acumulación de metales pesados en la parte aérea no fue muy alta. La explicación se encuentra en el hecho de que en los suelos contaminados de Aznalcóllar se acumulan concentraciones elevadas de varios metales con propiedades químicas (peso atómico, carga eléctrica) muy parecidas (Cu, Fe, Mn, Zn, Cd).

Estos últimos son absorbidos por las plantas a través del mismo mecanismo, resultando en una inhibición mutua en su asimilación. Algunas plantas, como por ejemplo la $B$. juncea, se comportó como una acumuladora de metales ( $\mathrm{Zn}$ y $\mathrm{Pb}$ ) y, por tanto, puede ser empleada como un indicador de la disponibilidad de estos elementos para las plantas en el suelo, y de la efectividad de los tratamientos de recuperación de suelos contaminados por metales pesados.

Por otra parte, la fitoextracción [11] también conocida como fitoacumulación, consiste en la absorción y translocación de los metales desde las raíces hasta las partes aéreas de las plantas, que posteriormente se cortarán y serán incineradas o bien acumuladas con el objetivo de reciclar los metales. Las plantas adecuadas para llevar a cabo acciones de este tipo deben cumplir algunas características como: 1. la tolerancia al metal que se desea eliminar (plantas hiperacumuladoras); 2. la acumulación, que se produzca fundamentalmente en la parte aérea de la planta y 3. que presenten un rápido crecimiento, así como una alta producción de biomasa en la parte aérea. En la actualidad se están efectuando investigaciones sobre la transferencia y mejora de los genes de estas plantas hiperacumuladoras, lo que hace muy interesante la continuación de este tipo de estudios. 
Hay 2 tipos de fitorremediación aplicables a los suelos contaminados por metales pesados: la fitoestabilización y la fitoextracción. La fitoestabilización se utiliza en los suelos donde la gran cantidad de contaminantes imposibilita la fitoextracción, se basa en el uso de plantas tolerantes a los metales para inmovilizar a través de su absorción y acumulación en las raíces o precipitación en la rizosfera, lo que reduce así su movilidad y su biodisponibilidad para otras plantas o microorganismos.

Un grupo de científicos del Servicio de Investigación Agrícola ARS [12], de la Universidad de Maryland, la Universidad de Melbourne en Australia y la Universidad Massey en Nueva Zelanda trabajaron con la planta 'alpine pennycress'. Los estudios concluyeron que esta planta tiene la capacidad de acumular cantidades vastas de cadmio y cinc en sus hojas y tallos por encima de la superficie del suelo. Al usar esta planta, cada año los granjeros podrían mover los metales del suelo a los brotes de la planta que se pueden cosechar, lo que les permitiría la reducción gradual de la concentración del cadmio en el suelo a niveles seguros.

La importancia de lo anterior radica primordialmente en el manejo de los residuos con contenido en metales pesados, ya que las alternativas de disposición son las que generalmente se aplican para residuos peligrosos: por ejemplo encapsulamiento, vitrificación o disposición en celdas de seguridad. Las cantidades por manejar resultan importantes con estos procedimientos, mientras que con los procesos de fitorremediación, al haber migrado los metales a la raíz o el tallo, los volúmenes manejados serán menores, con la consecuente reducción de costos en su manejo con las técnicas ya mencionadas para residuos peligrosos.

De otra parte, se verificó la importancia de las plantas acuáticas al utilizarlas como extractores de contaminantes a un bajo costo para depurar suelos, aguas contaminadas de tipo residual y domésticas. Cabe resaltar que para el montaje del sistema de fitorremediación mediante humedales se requiere un espacio apreciable y se debe realizar en zonas donde se conozca, por caracterizaciones previas, que los índices de contaminación sean importantes.

Se demostró que la zona de los rizomas es la que más acumula contaminantes; sin embargo es conveniente realizar el montaje de una unidad piloto donde se pueden controlar otras variables tales como $\mathrm{pH}$, salinidad, cantidad de nutrientes (controlando su migración con el fin de evitar la saturación de los cultivos), temperatura (mediante un efecto invernadero), toma de datos de propiedades en forma continua. Tales montajes deben ser observados durante periodos prolongados de fitorremediación con el propósito de elaborar un modelo matemático de diseño con el cual se pueda predecir el comportamiento de las plantas con el tiempo. Con ello, se buscaría dar a las plantas la periodicidad que se requiere para hacer más eficiente el proceso; así como implementar otro tipo de tratamientos donde, de acuerdo con los elementos que puede absorber la planta, se suministren tratamientos con sustratos químicos que cambien o combinen dichos elementos con el fin de permitir disminuir su reactividad y su volatilidad.

\section{Conclusiones y recomendaciones}

Dado que las 2 especies de plantas, Pteridium aquilinum (Cytheatae) y Biden leavis, se pueden denominar como acumuladoras de metales y, dependiendo del tipo de contaminante presente en el lodo a tratar, se recomienda montar un proceso de fitorremediación con varias especies sembradas para obtener las mejores eficiencias.

Se recomienda un estudio general, donde una de las variables a controlar sea el tiempo, ya que en algunos casos, al determinar las concentraciones, se pudo observar que después de cierto tiempo se presentaba colmatación y migración de contaminantes a los alrededores; es en ese momento cuando se debe suspender la adición de lodo al sistema.

Antes de aplicar la técnica de fitorremediación, al seleccionar las plantas empleadas, se deben tener en cuenta los procesos de migración de elementos que se puedan presentar en ellas, para evitar problemas de contaminación o acumulación de elementos no deseables en los lixiviados obtenidos y, por ende, la contaminación del suelo, acuíferos o aguas subterráneas.

Se corroboró que la fitorremediación se puede proponer como una técnica eficiente para el manejo 
de lodos procedentes de plantas de tratamiento de aguas residuales, toda vez que los resultados de los análisis químicos practicados a las partes principales de las especies vegetales (raíz, tallo y hojas) así lo demostraron.

Se demostró que la zona de los rizomas es la parte de la planta que más retiene contaminantes. Aunque el número de determinaciones analíticas fue apreciable, se considera pertinente realizar el montaje de la unidad piloto puesto que en ella, se podrán determinar inicialmente algunos algoritmos que conlleven a la generación de un modelo matemático de diseño bajo las condiciones actuales de Bogotá y sus alrededores. El sistema puede ser construido a un bajo costo (al compararlo con otros sistemas naturales ecotecnológicos como el landfarming que necesitan grandes áreas disponibles para el tratamiento con el riesgo de contaminar los suelos necesarios para la agricultura), ya que solo requiere la inversión inicial para el montaje del sistema y no demanda mano de obra especializada ni consumo de productos químicos.

Uno de los estudios más relevantes a plantear es concentrarse en la búsqueda de la relación raízmetal (o contaminante) y tallo grueso-metal (o contaminante) para identificar las especies vegetales que presentan el mayor porcentaje de absorción en estas zonas; este punto habrá de considerarse dado que, cuando ocurre la migración hacia las hojas y éstas caen al suelo o al agua, se reinicia el problema de contaminación en ellos, o en algunos casos se puede presentar su volatización, con la consecuente contaminación del aire.

Se comprobó que los tiempos del proceso de fitorremediación pueden ser muy prolongados, dado que análisis realizados a muestras tomadas en lapsos de 8 días, presentaron concentraciones con diferencias no apreciables.

El nivel de migración de contaminantes hacia las diferentes partes de las plantas deberá ser objeto de un estudio profundo, ya que de poderse emplear especies comestibles sería interesante verificar movilidad únicamente hacia la raíz y no hacia el tallo o las hojas. Con esto se mitigaría el gran problema que tienen las industrias provistas de plantas de tratamiento de efluentes, cual es el manejo de los lodos generados, a pesar de haberlos deshidratado.

El manejo de las partes contaminadas (hojas, tallos, raíces), luego de su colmatación por contaminantes, es otro objeto de estudio de interés sanitario. No obstante, las cantidades a manejar serían mucho menores a la de los lodos sin tratar, lo cual resulta interesante si se pretende realizar disposición con encapsulamiento, relleno de carreteras, o vitrificación, entre otros.

Si se comparan los resultados con otras experiencias, se ha demostrado que los árboles pueden ser modificados de forma que acumulen los contaminantes preferentemente en el tronco, no en las hojas. Según Ballester [13] se pueden cortar los troncos para usar su madera. Como los metales quedan atrapados en las células de la madera, ésta puede utilizarse sin riesgo.

Se debe resaltar que en el Instituto de Investigaciones Agrobiológicas de Galicia [3], se está trabajando la fitorremediación con árboles de tamaño considerable, los cuales, están siendo sometidos a un largo proceso de evaluación que incluye la experimentación en invernaderos, tal como se realizó el presente experimento, y en parcelas controladas con la autorización de la Comisión Nacional de Bioseguridad de esa región.

En Colombia, la investigación aún es incipiente, apenas se está evaluando la tecnología, por lo que es muy riesgoso pensar en aplicarla para residuos peligrosos por el momento. De ahí la necesidad de continuar con esto mediante el montaje de una unidad piloto de fitorremediación, donde se tengan en cuenta los parámetros ya mencionados, y de otra parte, se verifique que la migración sea hacia la parte de la raíz y en pequeña medida hacia el tronco (no hacia las hojas), previendo que en caso de que estos árboles puedan servir de soporte y alimento para aves silvestres no haya riesgo de contaminación a ellas. 


\section{Referencias Bibliográficas}

[1] Madejón P.“Elementos traza y nutrientes en álamo blanco tras el vertido tóxico de las minas deAznalcóllar". Investigación Agraria. Sistemas y Recursos Forestales.12 (3) 2003, pgs 19-32.

[2] Tiria L. "Manejo de lodos con contenido de cromo de una industria metalmecánica. Trabajo de grado en Ingeniería Ambiental y Sanitaria Universidad De La Salle, Colombia, 2002.

[3] Instituto de Investigaciones Agrobiológicas de Galicia y del Consejo Superior de Investigaciones Científicas (CSIC). En: publicación electrónica de la Oficina de Transferencia de Tecnología (OTT) y elaborado por la Unidad de Comunicación y Transferencia de Tecnología de la Delegación del CSIC en Cataluña. Junio de 2005.

[4] Cusato R; Tortosa N, Bartoloni. "Fitorremediacion: empleo de Discaria Americana". Facultad de Agro- nomía de la Universidad de Buenos Aires, 2002. CEQUIPE-INTI.

[5] Steven R. "Introduction to phytormediación". US, 2005, environmental Protection Agency. URL disponible en:http://www.epa.gov/nrmrVlrpod/rcb/introphy.html.

[6] Raskin I.. Phytoremediation of Toxic Metals: Using Plants to clean Up the Environment. John Wiley \& Sons, Inc. New York, 2000, pgs 53 y 93. URL disponible en:http:// www.ars.usda.gov/is/ar/archives/ jun00/ soil0600.html.

[7] Foroughbakhch R; Céspedes A; Alvarado M. "Aspectos ecológicos de los manglares y su potencial como fitorremediadores en el Golfo de México". Ciencia UANL, Universidad Autónoma de Nuevo León, México, 2004, abril-junio No.002.

[8] USDA, phytoremediación: Using Plants to clean Up Soils. Agricultural Research Service, United Sta- tes Department of Agriculture. USA, 2005.

[9] Agudelo B; Macias L; Suárez K. "Fitorremediación: la alternativa para absorber metales pesados de los biosólidos”. Red Revista Lasallista de Investigación, Colombia, 2009, pgs 5-11.

[10] Bernal, M; Bernal, M.P.; Clemente, R.; Vazquez, S.; Walker, D.J. "Aplicación de la fitorremediación a los suelos contaminados por metales pesados en Aznalcóllar". Asociación Española de Ecología Terres- tre Alicante, España. Ecosistemas, vol. 16, No.2, 2007, pgs. 1-14.

[11] Wong M. H. "Ecological restoration of mine degraded soils, with emphasis on metal contaminated soils". Chemosphere 50 (6), 2003, pgs 775- 778.

[12] Durham, Sh. Una planta delicada puede limpiar suelos contaminados por cadmio. Service of Investigation Agricola ARS. Beltsville, Maryland,2004.

[13] Ballester, A. Arboles en la fitorremediación de suelos y en la conservación de la Biodiversidad. España, Cataluña, 2005, 320 pgs. 